\title{
Ergodic Properties of a Kicked Damped Particle
}

\author{
Christian Beck ${ }^{\star}$ \\ Mathematics Institute, University of Warwick, Coventry CV4 7AL, UK
}

\begin{abstract}
We investigate a class of nonlinear dynamical systems describing the movement of a particle in a viscous medium under the influence of a kick force. These systems can be regarded as a generalization of the Langevin approach to Brownian motion in the sense that the fluctuating force on the particle is not Gaussian white noise but an arbitrary non-gaussian process generated by a nonlinear dynamical system. We investigate how certain properties of the force (periodicity, ergodicity, mixing property) transfer to the velocity of the particle. Moreover, the relaxation properties of the system are analysed.
\end{abstract}

\section{Introduction}

Consider a particle of mass 1 moving under the influence of the kick force

$$
L_{\tau}(t)=\sum_{n=0}^{\infty} \eta_{n} \delta(t-n \tau) .
$$

$\tau$ is the time difference between subsequent kicks (for simplicity we assume that the impulses are equidistant). $\eta_{n}$ is the strength of the kick at time $n \tau$. If the particle moves in a viscous medium, then in addition a friction force is exerted on the particle. We assume that this is proportional to the velocity $Y(t)$ of the particle. Thus the equation of motion is

$$
\dot{Y}=-\gamma Y+L_{\tau}(t)
$$

where $\gamma$ is the viscosity of the liquid. We will keep our considerations as general as possible and allow $Y(t)$ to take values in $\mathbf{R}^{m}$. Moreover, we assume that there is a discrete time dynamical system $T: X \rightarrow X \subset \mathbf{R}^{k}$ and a function $f: X \rightarrow \mathbf{R}^{m}$ such that

$$
\begin{aligned}
\eta_{n} & =f\left(x_{n-1}\right), \\
x_{n+1} & =T x_{n} .
\end{aligned}
$$

\footnotetext{
* Address after October 1, 1989: Institut für Theoretische Physik, RWTH, D-5100 Aachen, FRG
} 
That is to say, the evolution of the kick strengths is determined by the dynamical system $T$ on the phase space $X$ and projected onto $\mathbf{R}^{m}$ by the function $f{ }^{1}$

The solution $Y(t)$ of Eq. (2) can be presented as

$$
Y(t)=e^{-\gamma(t-n \tau)} y_{n}, \quad n=[t / \tau]
$$

([ ]: integer part) where $y_{n}$ is obtained by the recurrence relation

$$
U: \quad \begin{aligned}
x_{n+1} & =T x_{n} \\
y_{n+1} & =\lambda y_{n}+f\left(x_{n}\right)
\end{aligned}
$$

and $\lambda=e^{-\gamma \tau}$ is a parameter.

Our consideration implies that discrete-time dynamical systems of the form (6) have a direct "physical" interpretation: $y_{n}=Y(n \tau)$ is the velocity of a kicked damped particle. We assume that the initial velocity $y_{0}$ is fixed. If we also fix the starting value $x_{0}$, the time evolution of the velocity is deterministic for all times. Alternatively, $x_{0}$ can be regarded as a random variable with some probability distribution $\mu\left(x_{0}\right)$. Then $L_{\tau}(t)$ is per definitionem a stochastic process and Eq. (2) is a stochastic differential equation $[1,2,3]$. In fact, according to Kolmogorov's existence theorem [3] there exists for any stochastic process $\left(\eta_{n}\right)$ a phase space $X$, a dynamical system $T$, a map $f$ and a probability measure $\mu$ such that $\eta_{n}=$ $f\left(T^{n-1} x_{0}\right)$. In this sense the stochastic differential equation (2) and the dynamical system (6) are equivalent. However, we emphasize that Eq. (2) is much more general than the Langevin equation, because $L_{\tau}(t)$ is not uncorrelated Gaussian white noise but an arbitrary non-gaussian process uniquely determined by $T, f$ and $\mu$.

It appears to be interesting from both the mathematical and physical point of view to investigate the properties of dynamical systems of the form (6) in more detail. For the low dimensional case $k=m=1$, special examples have already been studied by several authors [4-8]. The first were Kaplan and Yorke [4]. The Kaplan Yorke map is obtained for the special choice $T x=2 x \bmod 1, f(x)=\cos 2 \pi x$. Later, Jensen and Oberman [5] studied the statistical properties of the system $T x=2 x \bmod 1, f(x)=\sin 2 \pi x$ and gave explicit results on correlation functions. Mayer and Roepstorf [6] investigated the system $T x=2 x^{2}-1, f(x)=x$ and further systems related to it. They proved the ergodicity of the systems, calculated the expectations of certain observables and pointed out the relation to Smale's solenoid [7]. Beck and Roepstorff [8] dealt with the limit $\lambda \rightarrow 1$. They showed that if $T$ has the so-called $\varphi$-mixing property and if $f$ is of bounded variation then the rescaled trajectory $\tau^{1 / 2} y_{n}$ generates the Ornstein-Uhlenbeck process in the scaling limit $\tau \rightarrow 0$. In fact, in this limit case there is an equivalence between the rescaled system (6) and the Langevin equation. In a recent paper Shimizu replaced the force $L_{\tau}(t)$ by its time integral version and considered the analogous problem [12].

In this paper we extend the discussion to arbitrary high phase space dimensions $k$ and $m$, and analyse the general properties of dynamical systems of the form (6). The physical motivation for this was pointed out in the introduction. We do not

\footnotetext{
1 In a physical picture $X$ may be regarded as the $10^{23}$-dimensional phase space of all the molecules in the liquid. Then $f$ projects down onto the velocity space of a single test particle
} 
choose special examples for $T$ and $f$, i.e. special kick forces but keep our considerations as general as possible. That is to say, we only assume that $T$ has certain properties and then investigate the consequences for the extended system $U$ determined by Eq. (6). It turns out that the most important properties of the $k$-dimensional system $T$ (existence of stable periodic orbits, ergodicity, mixing property) directly transfer to the $k+m$-dimensional system $U$. That is to say, if the $T$-dynamics is periodic (ergodic, mixing) the complete system $U$ has quite similar properties. A precise formulation is given in terms of Theorems 1-3. From the physical point of view these theorems describe how the properties of the force transfer to the velocity of the particle. This can be seen in analogy to the Langevin theory where it is well known that the statistical properties of the Wiener process are closely related to those of the Ornstein Uhlenbeck process. Our approach applies to a much wider class of dynamics generated by non-gaussian correlated kick forces.

Of special interest is the case of an exponentially mixing $T$-dynamics: We will show that if $T$ has relaxation time $\beta$ then the relaxation time of the $y$-variable of $U$ is given by $\max \left(\gamma^{-1}, \beta\right)$. In physical terms this means that the relaxation time of the particle's velocity is either determined by the inverse of the viscosity $\gamma$ of the liquid or by the relaxation time of the kick force, depending on which of the quantities is larger.

\section{Stable Periodic Orbits}

Firstly, we deal with the case of a periodic kick force. Suppose the dynamical system $T: X \rightarrow X$ has a stable periodic orbit. Does the extended system $U(x, y)=(T x, \lambda y+f(x))$ possess a stable periodic orbit, too? In fact, this is true for $|\lambda|<1$. The exact statement is as follows:

Theorem 1. Let $|\lambda|<1$ and $\left|y_{0}\right|<\infty$. Let $f: X \rightarrow \mathbf{R}^{m}$ be continuous and bounded $(|f|<B<\infty)$.

1. Let $T$ have a stable periodic orbit of length $L$ with basin of attraction $A \subset X$. Then $U$ has a stable periodic orbit of length $L$ with the same basin of attraction, i.e. for $x_{0} \in A, y_{0}$ arbitrary

$$
\lim _{n \rightarrow \infty}\left|y_{(n+1) L+j}-y_{n L+j}\right|=0, \quad j=1, \ldots, L .
$$

2. Let $T$ have an exponentially stable orbit, i.e. for $x_{0} \in A$,

$$
\left|x_{(n+1) L+j}-x_{n L+j}\right|<C \mu^{n} \quad 0<\mu<1
$$

(C: constant). Moreover, let $f$ be differentiable $\left(\left|f^{\prime}\right|<B<\infty\right)$ and let $\mu \neq|\lambda|^{L}$. Then for $x_{0} \in A, y_{0}$ arbitrary

$$
\left|y_{(n+1) L+j}-y_{n L+j}\right|<C_{1} \mu^{n}+C_{2}|\lambda|^{L n} \quad \forall n \in \mathbf{N}
$$

$\left(C_{i}\right.$ : constants). That is to say, $U$ has an exponentially stable orbit of length $L$ with the same basin of attraction as T. The approach to the periodic orbit is determined by $\max \left(\mu,|\lambda|^{L}\right)$.

3. For $\mu=|\lambda|^{L}$ one has $\left|y_{(n+1) L+j}-y_{n L+j}\right|<n C_{3} \mu^{n}$. 
Proof. 1) Iteration of (6) yields

$$
y_{n L+j}=\lambda^{n L+j} y_{0}+\sum_{k=0}^{n L+j-1} \lambda^{n L+j-1-k} f\left(x_{k}\right)
$$

Thus

$$
y_{(n+1) L+j}-y_{n L+j}=\lambda^{n L+j}\left(y_{L}-y_{0}\right)+\sum_{k=0}^{n L+j-1} \lambda^{n L+j-1-k}\left(f\left(x_{k+L}\right)-f\left(x_{k}\right)\right) .
$$
Splitting the sum $\sum_{k=0}^{n L+j-1}$ into two sums $\sum_{k=0}^{[n / 2] L}$ and $\sum_{k=[n / 2] L+1}^{n L+j-1}([]$ : integer part $)$ we
obtain

$$
\left|\sum_{k=0}^{n L+j-1} \lambda^{n L+j-1-k}\left(f\left(x_{k+L}\right)-f\left(x_{k}\right)\right)\right|<2 B \frac{|\lambda|^{n L / 2}}{1-|\lambda|}+\varepsilon \frac{1}{1-|\lambda|},
$$

where $\varepsilon \rightarrow 0$ for $n \rightarrow \infty$ provided $x_{0} \in A$. Moreover, from Eq. (7)

$$
\begin{aligned}
y_{L}-y_{0} & =\left(\lambda^{L}-1\right) y_{0}+\sum_{k=0}^{L-1} \lambda^{L-1-k} f\left(x_{k}\right) \\
& \Rightarrow\left|y_{L}-y_{0}\right|<\left|y_{0}\right|+\frac{1}{1-|\lambda|} B .
\end{aligned}
$$

Combining Eq. (8), (9), (10) we get $\left|y_{(n+1) L+j}-y_{n L+j}\right| \rightarrow 0$ for $n \rightarrow \infty$.

2) We write

$$
\left|y_{(n+1) L+j}-y_{n L+j}\right|<|\lambda|^{n L+j}\left|y_{L}-y_{0}\right|+\sum_{i=0}^{n}|\lambda|^{(n-i-1) L} \varepsilon_{i}
$$

where

$$
\varepsilon_{i}:=\sup _{j}\left|f\left(x_{(i+1) L+j-1}\right)-f\left(x_{i L+j-1}\right)\right|, \quad j=1, \ldots, L .
$$

Our assumption is $\left|x_{(i+1) L+j}-x_{i L+j}\right|<C \mu^{i}$ for all $j$. As $f$ is differentiable and $\left|f^{\prime}\right|<B<\infty$, the Taylor formula implies

$$
\left|f\left(x_{(i+1) L+j}\right)-f\left(x_{i L+j}\right)\right|<C B \mu^{i}
$$

Thus

$$
\begin{aligned}
\sum_{i=0}^{n}|\lambda|^{(n-i-1) L} \varepsilon_{i} & <\text { const }|\lambda|^{n L} \sum_{i=0}^{n}\left(\frac{\mu}{|\lambda|^{L}}\right)^{i+1} \\
& =\text { const }\left(\frac{\mu}{|\lambda|^{L}}-1\right)^{-1}\left(\frac{\mu^{n+2}}{|\lambda|^{2 L}}-\mu|\lambda|^{(n-1) L}\right)
\end{aligned}
$$

for $\mu \neq|\lambda|^{L}$. Inserting (14) and (10) into (11) we get

$$
\left|y_{(n+1) L+j}-y_{n L+j}\right|<C_{1}|\lambda|^{n L}+C_{2} \mu^{n} \text {. }
$$

3) Similarly we obtain for $\mu=|\lambda|^{L}$,

$$
\left|y_{(n+1) L+j}-y_{n L+j}\right|<C_{3} n|\lambda|^{n L} \text {. }
$$


Remark. It is easy to generalize the theorem for functions $f$ that are not differentiable but only obey a Hölder condition of type $|f(x+w)-f(x)|<$ const $|w|^{\alpha}, 0<\alpha<1$. In this case we have to replace $\mu$ by $\mu^{\alpha}$. The theorem is also valid for complex mappings $T$ and $U$ and complex $\lambda$ : The basins of attraction of $T$ and $U$ coincide.

Let us mention some further interesting consequences of the theorem: Choose for example for $T$ a 1-dimensional unimodal map such as $T x=1-a x^{2}$. As a consequence of the theorem, the higher dimensional system $U$ shows sub-harmonic bifurcations at the same parameter values $a$ as the system $T$, independent of $\lambda$. Both the accumulation point of bifurcations as well as the Feigenbaum constant $\delta$ are the same for both systems. Obviously the parameters $a$ and $\lambda$ are decoupled. Notice that for many other systems, for example those of Henon type rather than of Kaplan Yorke type, the parameters are not decoupled.

The physical interpretation of Theorem 1 can be summarized as follows: if the long time behaviour of the kick force is periodic, i.e. if $T$ possesses an attractive periodic orbit of length $L$, the particle's velocity will also become periodic with period $L$. The approach to the periodic orbit is determined by $\max \left(|\lambda|^{L}, \mu\right)$.

\section{Ergodicity}

We now deal with the case of an ergodic kick force. A dynamical system $T$ is called ergodic [9] if the time average of every continuous test function $\varphi$ exists and can be presented as an ensemble average:

$$
\lim _{J \rightarrow \infty} \frac{1}{J} \sum_{j=0}^{J-1} \varphi\left(x_{j}\right)=\int d \mu(x) \varphi(x)
$$

$\left(x_{j+1}=T x_{j}\right)$. Equation (17) must be valid for all starting values $x_{0}$ up to a set of measure 0 . The following theorem describes how the properties of the ergodic system $T$ transfer to the extended system $U(x, y)=(T x, \lambda y+f(x))$.

Theorem 2. Let $|\lambda|<1$ and $\left|y_{0}\right|<\infty$. Let $T: X \rightarrow X \subset \mathbf{R}^{k}$ and $f: X \rightarrow \mathbf{R}^{m}$ be continuous and bounded. Define functions $h_{n}: X \rightarrow \mathbf{R}^{m}$ by $h_{n}(x)=\sum_{j=0}^{n-1} \lambda^{n-1-j} f\left(T^{j} x\right)$.

1. Let $T$ be an ergodic dynamical system with invariant measure $\mu$. Then $U$ is also ergodic, in the following sense: For continuous and bounded observables $g$ and $n \in \mathbf{N}$

$$
\lim _{J \rightarrow \infty} \frac{1}{J} \sum_{j=0}^{J-1} g\left(x_{j}, y_{j}\right)=\int d \mu(x) g\left(T^{n} x, h_{n}(x)\right)+R_{n}
$$

with $\lim _{n \rightarrow \infty} R_{n}=0$.

2. If, in addition, $g$ is differentiable in the $y$-variable $(|(\partial / \partial y) g(x, y)|<B<\infty)$ then $R_{n}=O\left(|\lambda|^{n}\right)$, i.e. the rest term $R_{n}$ is exponentially small.

Proof. 1) Iteration of (6) yields

$$
y_{n}=\lambda^{n} y_{0}+\sum_{j=0}^{n-1} \lambda^{n-1-j} f\left(x_{j}\right)=\lambda^{n} y_{0}+h_{n}\left(x_{0}\right)
$$


Thus

$$
\left|y_{n}\right|<\left|y_{0}\right|+\frac{1}{1-|\lambda|} \sup _{x} f(x)<\infty
$$

Of course also $y_{n+j}=\lambda^{n} y_{j}+h_{n}\left(x_{j}\right)$. Thus for finite $n$

$$
\begin{aligned}
\lim _{J \rightarrow \infty} \frac{1}{J} \sum_{j=0}^{J-1} g\left(x_{j}, y_{j}\right) & =\lim _{J \rightarrow \infty} \frac{1}{J} \sum_{j=0}^{J-1} g\left(x_{j+n}, y_{j+n}\right) \\
& =\lim _{J \rightarrow \infty} \frac{1}{J} \sum_{j=0}^{J-1} g\left(T^{n} x_{j}, \lambda^{n} y_{j}+h_{n}\left(x_{j}\right)\right) .
\end{aligned}
$$

As $T$ and $f$ are continuous, $T^{n}$ and $h_{n}$ are also continuous. Moreover, $\lim \lambda^{n} y_{j}=0$ as $\left|y_{j}\right|$ is bounded. Due to our assumption $g$ is also continuous. Thus we can write

$$
\begin{aligned}
\lim _{J \rightarrow \infty} \frac{1}{J} \sum_{j=0}^{J-1} g\left(x_{j}, y_{j}\right) & =\lim _{J \rightarrow \infty} \frac{1}{J} \sum_{j=0}^{J-1}\left\{g\left(T^{n} x_{j}, h_{n}\left(x_{j}\right)\right)+R_{n}^{(j)}\right\} \\
& =\int d \mu(x) g\left(T^{n} x, h_{n}(x)\right)+\underbrace{\lim _{J \rightarrow \infty} \frac{1}{J} \sum_{j=0}^{J-1} R_{n}^{(j)}}_{R_{n}}
\end{aligned}
$$

with $\lim _{n \rightarrow \infty} R_{n}^{(j)}=0$ because of continuity of $g$. As $\left|R_{n}\right| \leqq \sup _{j}\left|R_{n}^{(j)}\right|$ we have $\lim _{n \rightarrow \infty} R_{n}=0$. 2) If $g$ is differentiable with regard to $y$, we can use Taylor's formula with finite rest term in (20). In this case

$$
R_{n}=\lim _{J \rightarrow \infty} \frac{1}{J} \sum_{j=0}^{J-1} \lambda^{n} y_{j} \frac{\partial}{\partial y} g\left(T^{n} x_{j}, \xi_{j}\right)
$$

for appropriate $\xi_{j}$. Thus

$$
\left|R_{n}\right|<|\lambda|^{n} \sup _{j}\left|y_{j}\right| \cdot B
$$

Remark. If $g$ satisfies a Hölder condition $|g(x, y+w)-g(x, y)|<$ const $|w|^{\alpha}$, $0<\alpha<1$, we get $R_{n}=O\left(|\lambda|^{\alpha n}\right)$.

Again, we would like to illustrate the "physical" meaning of Theorem 2. For this purpose we choose an observable $g$ that only depends on $y$. The theorem implies that if the kick force acting on the particle is ergodic, the particle's velocity is also ergodic. Moreover, the expectation of any observable $g$ can be calculated by evaluating $\int d \mu(x) g\left(T^{n} x, h_{n}(x)\right)$ for $n \rightarrow \infty$.

As a simple example let us consider the 2-dimensional dynamical system

$$
U: \begin{aligned}
& x_{j+1}=2 x_{j}^{2}-1, \\
& y_{j+1}=\lambda y_{j}+x_{j+1},
\end{aligned}
$$

i.e. $T(x)=f(x)=2 x^{2}-1\left(x_{0} \in X=[-1,1]\right)$. The system $T$ is ergodic (even mixing), the (natural) invariant measure is $d \mu(x)=\left(\pi \sqrt{1-x^{2}}\right)^{-1} d x . T$ is conjugated to a 


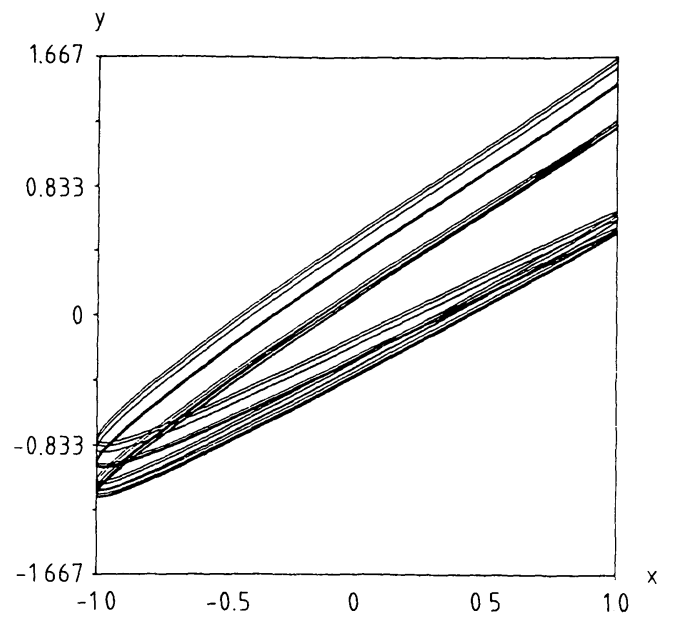

Fig. 1. Plot of the curve $A_{n}(u)=\left(\cos \pi 2^{n} u, \sum_{j=1}^{n} \lambda^{n-j} \cos \pi 2^{j} u\right), u \in[0,1], n=6, \lambda=0.4$

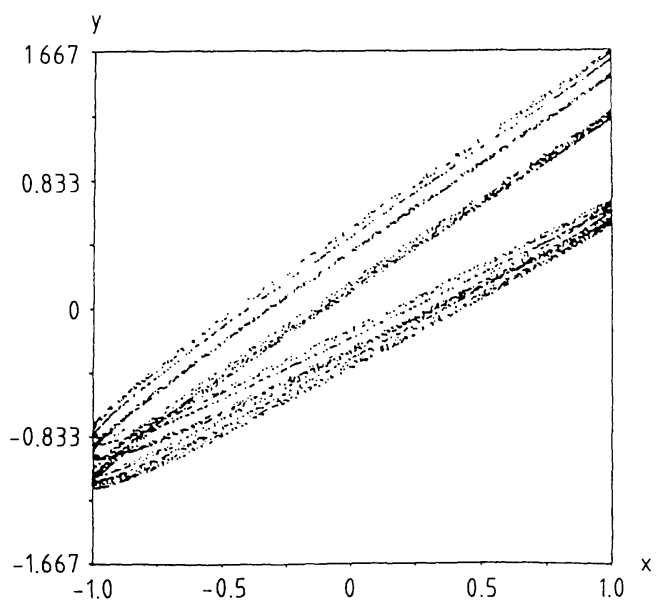

Fig. 2. Plot of 5000 iterates of the dynamical system $\left(x_{j+1}, y_{j+1}\right)=\left(2 x_{j}^{2}-1, \lambda y_{j}+x_{j+1}\right), \lambda=0.4$, for a single initial value $\left(x_{0}, y_{0}\right)$. The first iterates are omitted

Bernoulli shift [10]. Using the formula $2 \cos ^{2} \beta-1=\cos 2 \beta$ we can write

$$
x_{j}=\cos \pi 2^{j} u,
$$

where $u$ is a Lebesgue distributed random variable on $[0,1]$. The curve

$$
A_{n}(u)=\left(\cos \pi 2^{n} u, \sum_{j=1}^{n} \lambda^{n-j} \cos \pi 2^{j} u\right) \quad u \in[0,1]
$$

is the image of the ensemble of initial values after $n$ iterations of the map $U$. Expectations of observables $g(x, y)$ can be calculated as $\int_{0}^{1} d u g\left(\cos \pi 2^{n} u, \sum_{j=1}^{n} \lambda^{n-j} \cos \pi 2^{j} u\right)$ 
for $n \rightarrow \infty$. Figure 1 shows $A_{n}$ for $n=6, \lambda=0.4$. The curve coincides with the strange attractor of the map $U$ up to errors of $O\left(|\lambda|^{n}\right)$. In comparison Fig. 2 shows 5000 iterates of a single starting value. Whereas Fig. 1 represents an ensemble average, the dots in Fig. 2 can be regarded as a time average. Both figures coincide due to the ergodicity of the system.

\section{Mixing Properties}

In this section we make an even stronger assumption on the kick force: we assume it is mixing. A dynamical system $T$ is called mixing [9] if for all continuous functions $\varphi_{1}, \varphi_{2}$,

$$
\lim _{n \rightarrow \infty} C_{\varphi_{1}, \varphi_{2}}^{(T)}(n)=0 .
$$

Here $C_{\varphi_{1}, \varphi_{2}}^{(T)}(n)$ denotes the correlation function defined by

$$
\begin{aligned}
C_{\varphi_{1}, \varphi_{2}}^{(T)}(n)= & \left\{\lim _{J \rightarrow \infty} \frac{1}{J} \sum_{j=0}^{J-1} \varphi_{1}\left(x_{j+n}\right) \varphi_{2}\left(x_{j}\right)\right\}-\left\langle\varphi_{1}\right\rangle\left\langle\varphi_{2}\right\rangle, \\
& \left\langle\varphi_{i}\right\rangle=\lim _{J \rightarrow \infty} \frac{1}{J} \sum_{j=0}^{J-1} \varphi_{i}\left(x_{j}\right) \quad i=1,2,
\end{aligned}
$$

$\left(x_{j+1}=T x_{j}\right)$. If for $\varphi_{1}, \varphi_{2}$ in a function space $D$ the decay of the correlation function is exponentially fast, we call $T$ exponentially mixing with respect to $D$. We now show how the mixing properties of the map $T$ transfer to the system $U(x, y)=(T x, \lambda y+f(x))$.

Theorem 3. Let $|\lambda|<1$ and $\left|y_{0}\right|<\infty$. Let $T: X \rightarrow X \subset \mathbf{R}^{k}$ and $f: X \rightarrow \mathbf{R}^{m}$ be continuous and bounded.

1. Let $T$ be mixing. Then $U$ is also mixing, in the following sense: For continuous and bounded observables $g_{1}, g_{2}$,

$$
\lim _{n \rightarrow \infty} C_{g_{1}, g_{2}}^{(U)}(n)=0
$$

2. Let $T$ be exponentially mixing with respect to a function space $D$, i.e.

$$
\left|C_{\varphi_{1}, \varphi_{2}}^{(T)}(n)\right|<C \mu^{n}, \quad 0<\mu<1
$$

(C: constant) for $\varphi_{1}, \varphi_{2} \in D$ and $n \in \mathbf{N}$. Moreover, let $g_{1}, g_{2}$ be differentiable in the $y$-variable $\left(\left|(\partial / \partial y) g_{i}\right|<B<\infty\right)$ and assume that $g_{i}\left(T^{n}, h_{n}\right) \in D, i=1,2$. Then

$$
\left|C_{g_{1}, g_{2}}^{(U)}(n)\right|<C_{1} \mu^{n}+C_{2}|\lambda|^{n} \quad \forall n \in \mathbf{N}
$$

$\left(C_{i}\right.$ : constants). That is to say, the $m+k$-dimensional map $U$ is exponentially mixing. The relaxation time is determined by $\max (\mu,|\lambda|)$.

Proof. 1) First of all, Theorem 2 tells us that the averages

$$
\left\langle g_{i}\right\rangle=\lim _{J \rightarrow \infty} \frac{1}{J} \sum_{j=0}^{J-1} g_{i}\left(x_{j}, y_{j}\right) \quad i=1,2
$$


exist. Without loss of generality we assume that they vanish. For finite $n \in \mathbf{N}$ one has

$$
\begin{aligned}
C_{g_{1}, g_{2}}^{(U)}(n) & =\lim _{J \rightarrow \infty} \frac{1}{J} \sum_{j=0}^{J-1} g_{1}\left(x_{j+n}, y_{j+n}\right) g_{2}\left(x_{j}, y_{j}\right) \\
& =\lim _{J \rightarrow \infty} \frac{1}{J} \sum_{j=0}^{J-1} g_{1}\left(x_{j+2 n}, y_{j+2 n}\right) g_{2}\left(x_{j+n}, y_{j+n}\right) \\
& =\lim _{J \rightarrow \infty} \frac{1}{J} \sum_{j=0}^{J-1} g_{1}\left(T^{n} x_{j+n}, \lambda^{n} y_{j+n}+h_{n}\left(x_{j+n}\right)\right) g_{2}\left(T^{n} x_{j}, \lambda^{n} y_{j}+h_{n}\left(x_{j}\right)\right) .
\end{aligned}
$$

In analogy to the proof of Theorem 2 we can write

$$
C_{g_{1}, g_{2}}^{(U)}(n)=C_{g_{1}\left(T^{n}, h_{n}\right), g_{2}\left(T^{n}, h_{n}\right)}^{(T)}(n)+R_{n}
$$

with $\lim R_{n}=0$. By assumption the functions $g_{i}\left(T^{n} x, h_{n}(x)\right), i=1,2$ continuously $n \rightarrow \infty$ depend on $x$. Thus the mixing property of $T$ implies

$$
\lim _{n \rightarrow \infty} C_{g_{1}\left(T^{n}, h_{n}\right), g_{2}\left(T^{n}, h_{n}\right)}^{(T)}(n)=0,
$$

and therefore $\lim _{n \rightarrow \infty} C_{g_{1}, g_{2}}^{(U)}(n)=0$.

2) If $g_{1}$ and $g_{2}$ are differentiable with respect to $y$ we can apply Taylor's formula to Eq. (31):

$$
g_{1}\left(T^{n} x_{j+n}, \lambda^{n} y_{j+n}+h_{n}\left(x_{j+n}\right)\right)=g_{1}\left(T^{n} x_{j+n}, h_{n}\left(x_{j+n}\right)\right)+\lambda^{n} y_{j+n} \frac{\partial}{\partial y} g_{1}\left(T^{n} x_{j+n}, \xi_{j}\right)
$$

( $g_{2}$ analogous). It follows

$$
\left|C_{g_{1}, g_{2}}^{(U)}(n)\right|<\left|C_{g_{1}\left(T^{n}, h_{n}\right), g_{2}\left(T^{n}, h_{n}\right)}^{(T)}(n)\right|+2 \sup _{j}\left|y_{j}\right| \cdot B^{2} \cdot|\lambda|^{n}+O\left(|\lambda|^{2 n}\right) .
$$

As $g_{i}\left(T^{n}, h_{n}\right) \in D, i=1,2$ we arrive at

$$
\left|C_{g_{1}, g_{2}}^{(U)}(n)\right|<C_{1} \mu^{n}+C_{2}|\lambda|^{n}
$$

Remark. For Hölder continuous observables with $\left|g_{i}(x, y+w)-g_{i}(x, y)\right|$ $<$ const $|w|^{\alpha}$ we get $\left|C_{g_{1}, g_{2}}^{(U)}(n)\right|<C_{1} \mu^{n}+C_{2}|\lambda|^{\alpha n}$.

Again let us interprete Theorem 3 in a physics-related way: If the kick force is mixing, the velocity of the particle is also mixing. In case of an exponentially mixing kick force the approach to equilibrium is determined by either $\mu$ or $|\lambda|$, depending on which of the quantities is larger. It is interesting to see how the relaxation behaviour depends on the class of observables considered: Correlations for differentiable observables fall off like $|\lambda|^{n}$ provided $\mu<|\lambda|$. Because of $\lambda=e^{-\gamma \tau}$ and $t=n \tau$, we get the relaxation time $\gamma^{-1}$, which is in fact the relaxation time we would expect looking at Eq. (2). However, if we choose Hölder continuous observables, the relaxation time is larger, namely $(\alpha \gamma)^{-1}$ which seems to be "unphysical." If $g$ is only continuous there may be no exponential decay at all. In general we learn that relaxation times are intimately related to the properties of 
the corresponding observables [11]. It is a non-trivial problem to decide which kind of observables a physicist should choose in order to get a relaxation time of physical significance. Our consideration strongly suggests that differentiable observables are the right candidates. This coincides with Bowen's definition of observables [9].

\section{References}

1. van Kampen, N. G.: Stochastic processes in physics and chemistry. Amsterdam: North-Holland 1981

2. Schuss, Z:: Theory and applications of stochastic differential equations. New York: Wiley 1980

3. Friedman, A.: Stochastic differential equations and applications. New York: Academic Press 1975

4. Kaplan, J. L., Yorke, J. A.: Lecture Notes in Mathematics vol. 730, p. 204. Berlin, Heidelberg, New York: Springer 1979

5. Jensen, R. V., Oberman, C. R.: Phys. Rev. Lett. 46, 1547 (1981)

6. Mayer, D., Roepstorff, G.: J. Stat. Phys. 31, 309 (1983)

7. Smale, S.: Lecture Notes in Mathematics vol. 615, p. 48. Berlin, Heidelberg, New York: Springer 1977

8. Beck, C., Roepstorff, G.: Physica 145A, 1 (1987)

9. Bowen, R.: Lecture Notes in Mathematics vol. 615, p. 117. Berlin, Heidelberg, New York: Springer 1977

10. Ulam, S. M., von Neumann, J.: Bull. Am. Math. Soc. 53, 1120 (1947)

11. Mayer, D., Roepstorff, G.: J. Stat. Phys. 47, 149 (1987); 50, 331 (1988)

12. Shimizu, T.: Phys. Lett. 140A, 343 (1989)

Communicated by J.-P. Eckmann

Received January 31, 1989; in revised form November 11, 1989 